\title{
INTEGRAL EQUATIONS AND ACTUARIAL RISK MANAGEMENT: SOME MODELS AND NUMERICS
}

\author{
A. MAKROGLOU
}

Dept. of Mathematics, Univ. of Portsmouth

1st Floor, Buckingham Building, Lion Terrace

Portsmouth, Hampshire PO1 3HE, UK

E-mail: athena.makroglou@port.ac.uk

Received 31 December 2002

\begin{abstract}
The problem of the estimation of the probability $R(z, t)$ (here $t$ is time, $z$ is initial reserve) of the finite time non-ruin problem for a risk business such as an insurance company is considered, with respect to:

- presenting models that have been used in the literature in the form of integral / integrodifferential equations,

- reviewing some analytical and computational methods used for their solution,

- presenting numerical results obtained with one method (a global Lagrange type approximation in the $z$ - space).
\end{abstract}

Key words: Partial Volterra integro-differential equations, first order, numerical solution, global Lagrange type approximations, actuarial risk management, finite time collective nonruin

\section{INTRODUCTION}

The problem of estimating the probability of non-ruin given an initial reserve is very important in modelling a risk business such as an insurance company. In the case of finite time non-ruin, we are concerned with the estimation of the probability

$$
R(z, t)=P[Z(s)>0,0 \leq s \leq t \mid Z(0)=z]
$$

where, using the notation of Knessl and Peters [16], $Z(s)$ denotes the risk reserve at time $s$ and $z$ is the initial risk reserve.

The simple model of the risk business of an insurance company considered, 
assumes that:

Risk reserve $=$ initial reserve + total premiums - total claims.

Claim sizes at time $t$ denoted by $X_{t}$ are assumed to have distribution function $B(x)=P\left[X_{t} \leq x\right]$ with corresponding density function $b(x)$ and are assumed to arrive according to a Poisson process $N_{t}$ with parameter $\lambda$. This means that

$$
p_{n}(t)=P\left[N_{t}=n\right]=\frac{e^{-\lambda t}(\lambda t)^{n}}{n !}, \quad n=0,1, \ldots
$$

Then $S_{t}=\sum_{i=1}^{N_{t}} X_{t_{i}}$, the accumulated claims process is a compound Poisson process. It is also assumed that the premium is received at a continuous rate $\beta(r)$ which depends on the current reserve $Z(t)=r$. In the absence of claims, it is assumed that the reserve satisfies the deterministic equation

$$
\frac{d Z(t)}{d t}=\beta(Z(t))
$$

The finite time models of collective non-ruin are usually described by partial Volterra integro-differential equations (PVIDEs). There are models in the form of integral equations too. This problem has been solved by a number of analytical and numerical approaches for special cases of $\beta(Z(t))$ and $B(x)$. These approaches often involve use of Laplace transforms and analytical or numerical inversion (cf. Seal [26; 27], Peters and Mangel [24], and Knessl and Peters $[15 ; 16])$. Some of the results of Seal $[26]$ and Knessl and Peters [15] were verified by Harper $[9 ; 10]$.

The approaches (Seal $[26]$ and Knessl and Peters $[15 ; 16]$ ) depend on the ability to solve analytically the equation for the Laplace transform of the unknown function $R(z, t)$. For more general choices of $B(x)$ and $\beta(Z(t))$ the analytical solution of the equation satisfied by the Laplace transform of $R(z, t)$ might not be always possible. Even for simple choices of $B(x)$ and $\beta(Z(t))$, the form of the resulting formulae is often complicated.

Thus, Makroglou [18; 20] followed an approach that combines the numerical solution of the corresponding VIDE satisfied by the Laplace transform of the unknown function $R(z, t)$ and numerical Laplace transform inversion. In another paper Makroglou [19] explored the idea of solving directly the PVIDE satisfied by $R(z, t)$ by the use of multiquadric approximations applied to this problem in the fashion of Bonzani [3].

Solving the PVIDEs numerically is very important since it allows easy extensions to the treatment of the problem under more complicated assumptions for $B(x)$ and $\beta(Z(t))$ when analytical solutions are difficult to obtain. 
In this paper we review some analytical and computational methods used for solving such models and we present some numerical results obtained by implementing a new method also based on the work of Bonzani [3] for partial differential equations. This method uses global polynomial approximations of the unknown function $R(z, t)$ in the $z$-space combined with a ordinary differential equation solver.

The organization of the paper is as follows: In Section 2 the form of the PVIDE of the problem of finite time non-ruin is stated, together with some model details and references to integral equation models. In Section 3, a brief review of existing analytical and computational methods is given. In Section 4, the description of the new method (LAG-ODE) for solving the PVIDE is presented and in Section 5 some numerical results are given. Finally conclusions and ideas for further research may be found in Section 6 .

\section{INTEGRAL AND INTEGRO-DIFFERENTIAL EQUATION MODELS}

In this Section the form of the PVIDE satisfied by $R(z, t)$ is given (Section 2.1 ), together with some model details (Section 2.2). References and some details on integral equation models are given in Section 2.3.

\subsection{The PVIDE model}

Using the assumptions stated in the Introduction and using probabilistic arguments, a PVIDE model is derived for the probability $R(z, t)$, see for example Arfwedson [2], Peters and Mangel [24], Knessl and Peters [15; 16] and Grandell [8] for more details. It has the following form

$$
\frac{\partial R(z, t)}{\partial t}=-\lambda R(z, t)+\beta(z) \frac{\partial R(z, t)}{\partial z}+\lambda \int_{0}^{z} R(z-y, t) d B(y),
$$

with conditions

$$
\lim _{z \rightarrow \infty} R(z, t)=1, \quad R(z, 0)=1, z \geq 0 .
$$

We also have that $R(z, t)=0, z<0$.

\subsection{Some model details}

Most of the methods applied for solving the IDEs (2.1) - (2.2) treat special cases of the problem concerning the choice of the model for the premiums $\beta(Z(t))$, and the distribution function $B(x)$. The choices for the premium function are:

$$
\beta(Z(t))=\beta_{0}=\text { const }
$$


and

$$
\beta(Z(t))=\beta_{0}+\gamma Z(t)
$$

where $\beta_{0}$ denotes the rate (constant) at which the premiums come in, and $\gamma$ is the interest rate that applies to risk reserve until a claim is made.

The choices of the distribution function $B(x)$ and its density function $b(x)$, include the Gamma distribution with $v=1$ (exponential) and $v=2$.

\subsection{Some integral equation models}

Integral equations have also been used. Some recent papers include: Usabel [28], Lin and Willmot [17], Jasiulewicz [12], Yang and Zhang [29], Brekelmans and Waegenaere [4], Dickon and Waters [6].

\section{DESCRIPTION OF SOME EXISTING METHODS}

In this section an overview of some analytical and computational methods for solving the PVIDE (2.1) - (2.2) is given (Sections 3.1 and 3.2 respectively).

\subsection{Analytical methods of solution of the PVIDE model}

Various techniques exist which include:

- Use of Laplace transforms (cf. Seal [26; 27], Knessl and Peters [15; 16], De Vylder and Goovaerts [5], Pervozvansky [23].

- Expansion of $R(z, t)$ as a gamma series (cf. Albrecher, Teugels, and Tichy [1]).

- Use of inequalities in the form of upper and lower bounds for $R(z, t)$, and use of recurrence relations (cf. Ignatov, Kaishev, Krachunov [11]).

More references may be found in the recent survey by Paulsen [22] for ruin theory with compounding assets.

In this section a brief description of one method which has been used in the literature for the solution of $(2.1)-(2.2)$ is given for introductory purposes (see also Makroglou [20]): Knessl's and Peter's method (Knessl and Peters [15]) in Section 3.1.1. Some of the results of this paper have been verified in detail in Harper $[9 ; 10]$. The method has also been used for comparison purposes in the presentation of numerical results in Makroglou [20].

\subsubsection{Approach of Knessl and Peters}

The approach of Knessl and Peters [15] can be summarized as follows: they consider the Laplace transform $Q(z, s)$ of the function $R(z, t)$ over time:

$$
Q(z, s)=\int_{0}^{\infty} e^{-s t} R(z, t) d t
$$


Then multiplying both sides of equation (2.1) by $e^{-s t}$ and integrating from 0 to $\infty$ with respect to $t$ one obtains the following linear integro-differential equation

$$
s Q(z, s)-1=\beta(z) \frac{\partial Q(z, s)}{\partial z}-\lambda Q(z, s)+\lambda \int_{0}^{z} Q(z-y, s) d B(y)
$$

with the boundary condition

$$
Q(\infty, s)=\frac{1}{s}
$$

They solve this equation analytically by conversion to a second order ordinary differential equation for the special case that $B(x)$ is the exponential distribution $\left(B^{\prime}(x)=b(x)=\alpha e^{-\alpha x}, \mu=\frac{1}{\alpha}, \mu\right.$ the mean). Analytical inversion of $Q(z, s)$ is then applied to obtain $R(z, t)$. Both cases (2.3) and (2.4) for the premiums are considered. Exact and asymptotic solutions are derived for case (2.3) and exact ones for the case (2.4). Asymptotic solutions for the case (2.4) were given in Knessl and Peters [16]. Some of the formulae are complicated and involve series and integrals. Numerical results which are obtained by numerical evaluation of the final formulae for $R(z, t)$ using MATHEMATICA can be found in Knessl and Peters [16].

The analytical (exact) solutions provided in Knessl and Peters [15] are used for testing purposes and computation of the true errors. These are stated for convenience in Section 5 (Numerical results).

\subsection{Computational methods for the PVIDE model}

Computational methods for solving the PVIDE model (2.1) - (2.2) fall into two main categories:

(i) Solve (analytically or numerically) the equation satisfied by the Laplace transform of $R(z, t)$ with respect to $z$ or $t$ and then invert the Laplace transform to find $R(z, t)$ using an analytical or a numerical inversion method, or a combination of the two.

(ii) Solve the PVIDE satisfied by $R(z, t)$ directly to find estimates of $R(z, t)$ at various $z, t$ values.

Methods of type (i) include:

- Seal [26] who used Gauss type Laplace inversion methods combined with numerical integration rules to evaluate $R(z, t)$. An expression for $R(z, t)$ was produced by applying analytical inversion methods to a formula representing the analytical solution of the (partial) differential equation satisfied by the Laplace transform of $R(z, t)$ with respect to $z$.

- Knessl and Peters [15; 16] who found a VIDE (3.1) - (3.2) satisfied by the Laplace transform $Q(z, s)$ of $R(z, t)$ with respect to $t$, solved this equation 
analytically to find an expression for $Q(z, s)$ and then inverted $Q(z, s)$ also analytically to find an expression for $R(z, t)$. The numerical results were obtained by numerically (Mathematica) evaluating the expression for $R(z, t)$.

- Usabel [28] who used a Gaver-Stehfest Laplace inversion technique applied to an integral equation satisfied by the ruin probability.

- Makroglou [18; 20] who used the VIDE (3.1) for the Laplace transform of $R(z, t)$ with respect to $t$ according to the Knessl and Peters [15] approach and solved this equation by a numerical method which combines polynomial collocation methods for solving the VIDE and numerical Laplace transform inversion by the ACM routine 619 for obtaining estimates of $R(z, t)$ at $z, t$ values.

Methods of type (ii) include:

- Makroglou [19] who extended a method applied to partial differential equations by Bonzani [3] for solving directly the PVIDE (2.1) - (2.2) using multiquadric approximations in the $z$-space combined with an ODE solver.

Other approaches for estimating the probability $R(z, t)$ of non-ruin exist in the literature, as for example through the use of recursive formulae or evaluation of upper and lower bounds (cf. Ignatov, Kaishev and Krachunov [11] and the references therein).

For detailed description of these computational methods we refer to the original papers.

In the next Section we present a numerical method for solving the PVIDE model $(2.1)-(2.2)$ by a global Lagrange type polynomial approximation method combined with an ODE solver, (LAG-ODE method) which is based on a numerical approach applied to partial differential equations by Bonzani [3].

\section{LAG-ODE METHOD}

We start from equation $(2.1)-(2.2)$ rewritten as:

$$
\begin{aligned}
\frac{\partial R(z, t)}{\partial t} & =-\lambda R(z, t)+\beta(z) \frac{\partial R(z, t)}{\partial z}-\lambda \int_{0}^{z} b(z-y) R(y, t) d y \\
R(\infty, t) & =1, t \geq 0, \quad R(z, 0)=1, z \geq 0
\end{aligned}
$$

We are going to use Lagrange type global approximations for the numerical solution of (4.1) - (4.2) following the approach of Bonzani [3] who applied sinc and Lagrange type approximations to second order semilinear partial differential equations in one space dimension.

For fixed $t$, the following approximation (cf. Bonzani [3], Kansa [13; 14]) to 
Integral equations and actuarial risk management: models and numerics 149 the unknown function $R(z, t)$ of equation (4.1) is used:

$$
\begin{aligned}
& R(z, t)=\sum_{j=1}^{n+1} a_{j}(t) L_{j}(t), \quad t \geq 0,0 \leq z \leq \bar{z} \\
& L_{j}(z)=\prod_{k=1, k \neq j}^{n+1} \frac{\left(z-z_{k}\right)}{\left(z_{j}-z_{k}\right)}, 0=z_{1}<\ldots<z_{n}<z_{n+1}=\tilde{z}
\end{aligned}
$$

The boundary condition $g(t)=R(\tilde{z}, t)$ was found using the true solution. This boundary condition was used to express $a_{n+1}(t)$ in (4.3) in terms of $a_{1}(t), \ldots, a_{n}(t)$, giving (since $\left.L_{n+1}\left(z_{n+1}\right)=1\right)$

$$
a_{n+1}(t)=g(t)-\sum_{j=1}^{n} a_{j}(t) L_{j}(\tilde{z})
$$

In practice, a $\tilde{z}$ value has to be chosen to represent the $\infty$, so that the given boundary condition $\lim _{z \rightarrow \infty} R(z, t)=1$ can be used instead.

Using (4.5) in (4.3) we obtain

$$
R(z, t)=\sum_{j=1}^{n} a_{j}(t) L_{j}(z)+L_{n+1}(z)\left[g(t)-\sum_{j=1}^{n} a_{j}(t) L_{j}(\tilde{z})\right]
$$

or

$$
R(z, t)=\sum_{j=1}^{n} a_{j}(t) P_{j}(z)+\Phi(z, t)
$$

where

$$
\begin{aligned}
P_{j}(z) & =L_{j}(z)-L_{n+1}(z) L_{j}(\tilde{z}), \quad j=1, \ldots, n \\
\Phi(z, t) & =g(t) L_{n+1}(z) .
\end{aligned}
$$

We may note that with $L_{j}(z)$ given by (4.4), $P_{j}(z)$ in (4.7) simplifies to $P_{j}(z)=L_{j}(z)$. From (4.6) we then obtain

$$
\begin{aligned}
& \frac{\partial R(z, t)}{\partial t}=\sum_{j=1}^{n} \frac{d a_{j}(t)}{d t} P_{j}(z)+\frac{\partial \Phi(z, t)}{\partial t} \\
& \frac{\partial R(z, t)}{\partial z}=\sum_{j=1}^{n} a_{j}(t) \frac{d P_{j}(z)}{d z}+\frac{\partial \Phi(z, t)}{\partial z} .
\end{aligned}
$$


Using now (4.6), (4.8), (4.9) in equation (4.1) and collocating at $z=z_{k}, k=$ $1, \ldots, n$ we find that the coefficients $a_{j}(t)$ in (4.6) satisfy a first order system of ordinary differential equations of the form

$$
\frac{d \mathbf{a}(t)}{d t}=S^{-1} F \mathbf{a}+S^{-1} \mathbf{b}(t)
$$

where $S, F$ are $n \times n$ matrices with elements

$$
\begin{aligned}
& S_{k j}=P_{j}\left(z_{k}\right), \quad k, j=1,2, \ldots, n, \\
& F_{k j}=-\lambda P_{j}\left(z_{k}\right)+\beta\left(z_{k}\right) \frac{d P_{j}\left(z_{k}\right)}{d z}+\lambda J_{j}\left(z_{k}\right)
\end{aligned}
$$

and $\mathbf{b}(t)$ is given by

$$
\left.b_{k}(t)=-\frac{\partial \Phi\left(z_{k}, t\right)}{\partial t}-\lambda \Phi\left(z_{k}, t\right)+\beta\left(z_{k}\right) \frac{\partial \Phi\left(z_{k}, t\right)}{\partial z}+\lambda I\left(z_{k}, t\right)\right),
$$

where $J_{j}\left(z_{k}\right), I\left(z_{k}, t\right)$ are approximations to the integrals

$$
\int_{0}^{z_{k}} b\left(z_{k}-y\right) P_{j}(y) d y, \quad \int_{0}^{z_{k}} b\left(z_{k}-y\right) \Phi(y, t) d y
$$

respectively by a quadrature rule, and $P_{j}(z), \Phi(z, t)$ are given by (4.7).

To solve the ODE system (4.10) we also need starting values $\mathbf{a}(0)$. These are found using the second of the conditions (4.2) for $R$, that is $R(z, 0)=1, z \geq 0$ and (4.6). We thus obtain the linear system of equations in $a_{j}(0), j=1, \ldots, n$ :

$$
\sum_{j=1}^{n} a_{j}(0) P_{j}\left(z_{k}\right)=1-\Phi\left(z_{k}, 0\right), \quad k=1,2, \ldots, n .
$$

After we solve the ODE system (4.10) for the values of $t$ we need, the function $R(z, t)$ is evaluated from (4.6). We may note that values $R\left(z_{k}, t\right)$ at the mesh points $z_{k}, k=1, \ldots, n$ are equal to $a_{k}(t)$.

For other methods applied to hyperbolic partial differential equations of higher order which include error estimates (but no numerical results) we refer for example to Yanik and Fairweather [30], Pani, Thomée and Wahlbin [21], Fairweather [7].

\section{NUMERICAL RESULTS}

Numerical results are presented for the LAG-ODE method presented in Section 4. Particular coefficients and data used: 


$$
b(x)=\alpha e^{-\alpha x}
$$

and the case $\beta(z)=\beta_{0}+\gamma z$ (case 2.4), with the true solution given (Knessl and Peters [15], verified also by Harper [10]) as: (variable $\beta$ case, for $\lambda=\gamma$ )

$$
\begin{aligned}
& Q(z, s)=\frac{1}{s}-\frac{\gamma}{s\left(s+\gamma+\alpha \beta_{0}\right)} e^{-\alpha z} \\
& R(z, t)=1-\frac{\gamma}{\gamma+\alpha \beta_{0}} e^{-\alpha z}\left(1-e^{-\left(\gamma+\alpha \beta_{0}\right) t}\right) .
\end{aligned}
$$

The general case $(\lambda \neq \gamma)$ is also treated in Knessl and Peters [15] who obtained a form for $R(z, t)$ in terms of hypergeometric functions. Asymptotic estimates for $R(z, t)$ for the cases $\lambda>\alpha \beta_{0}, \lambda<\alpha \beta_{0}$ and $\lambda \sim \alpha \beta_{0}$ are given in Knessl and Peters [16].

Some numerical results for $R(z, t)$ using the LAG-ODE method of Section 4 are presented in Table 2. Comparisons are made with the COL-LAP method of Makroglou [20] (Table 1).

\section{Computational details:}

a) For the numerical inversion of Laplace transforms, the ACM routine 619 (Piessens and Huysmanns [25]) was used.

b) The COL-LAP method (Makroglou [20]) was used with collocation parameters $c_{1}=0.0, c_{2}=1.0$ which makes the collocation method for solving for $Q(z, s)$ the Laplace transform of $R(z, t)$ with respect to $t$, identical to the trapezoidal method.

c) The LAG-ODE method (equations (4.10) - (4.11)) was used with $n=10$ in the range $z \in[0,3]$ and collocation points $z_{k}, k=1, \ldots, n+1$ the Chebyshev points which in the range $[a, b]$ are given by :

$$
\begin{aligned}
& z_{k}=x_{k} \frac{(b-a)}{2}+\frac{(a+b)}{2}, \quad k=1,2, \ldots, n, \\
& x_{k}=\cos \frac{2 k-1}{2 n} \pi, \quad k=1,2, \ldots, n .
\end{aligned}
$$

The ODE numerical solver used for the solution of the system of ordinary differential equations in $a_{j}(t)$, was the routine ODE23s of Matlab5. The integrals were approximated by a composite trapezoidal rule with $N_{T}=80$ nodes. Results obtained using the Matlab5 routine QUAD based on an adaptive implementation of Simpson's rule, gave similar results. For small 
values of $z_{k}$, some computations with a smaller value of $N_{T}$ resulted in improved results. So for example, for $t=1$ and $z=2$ the computed value for $R(z, t)$ for the LAG-ODE method was equal to 0.9571205 with true absolute error equal to $0.14 D-4$.

d) The following tolerances were used: Tol1=1.D-10 for the solution of the collocation systems of the method COL-LAP treated as nonlinear, so that to have a general program which works for both linear and nonlinear equations. Tol2 =1.D- 4 with the ACM 619 routine for the numerical inversion of Laplace transforms. Tol3=1.D-5 with the ODE23s routine of Matlab5.

e) The values of the parameters $\alpha, \beta, \beta_{0}, \gamma, \lambda$ are as denoted in the tables and they correspond to the case $\lambda<\alpha \beta_{0}$ of Knessl and Peters [15; 16].

Table 1.

Variable $\beta(z)$ case (2.4), COL-LAP method, results for $R(2, t) \lambda=\gamma=1, \beta_{0}=2, \alpha=1$

\begin{tabular}{cccccc}
\hline$t$ & Exact & Computed, N $=40$ & Abs. error & Computed, $N=80$ & Abs. error \\
1.0 & 0.9571342 & 0.9571244 & $0.98 D-5$ & 0.9571341 & $0.16 D-6$ \\
3.0 & 0.9548938 & 0.9548852 & $0.86 D-5$ & 0.9548940 & $0.17 D-6$ \\
5.0 & 0.9548883 & 0.9548796 & $0.86 D-5$ & 0.9548884 & $0.16 D-6$ \\
10.0 & 0.9548882 & 0.9548796 & $0.86 D-5$ & 0.9548884 & $0.16 D-6$ \\
\hline
\end{tabular}

Table 2.

Variable $\beta$ case(2.4), LAG-ODE method, results for $R(2, t)$, $\lambda=1, \gamma=1, \beta=2, \alpha=1, n=10, N_{T}=80$

\begin{tabular}{cccc}
\hline$t$ & Exact & Computed & Abs. error \\
\hline 1.0 & 0.9571342 & 0.9571360 & $0.18 D-5$ \\
3.0 & 0.9548938 & 0.9549112 & $0.17 D-4$ \\
5.0 & 0.9548883 & 0.9549059 & $0.18 D-4$ \\
10.0 & 0.9548882 & 0.9549059 & $0.18 D-4$ \\
\hline
\end{tabular}

\section{CONCLUSIONS}

The estimation of the probability of finite time non-ruin $R(z, t)$ for the variable $\beta(Z(t)$ ) (case (2.4)), was obtained by solving the governing PVIDE by a fully numerical approach, (LAG-ODE) which solves numerically the PVIDE directly by a global Lagrange type approximation method and numerical solution of the resulting system of ordinary differential equations. Comparisons are made with the COL-LAP method of Makroglou [20] (Table 1).

Comparing the results of Table 2 (LAG-ODE method) with those of Table 1 (COL-LAP method) we may note that the former appear to be less accurate than the latter. The LAG-ODE results could improve further if used with a larger value of $n$ ( $n+1$ is the number of collocation points). 
Computations with the Knessl and Peters [15] approach implemented by inverting numerically the exact form of the Laplace transform $Q(z, s)$ (section 3.1.1), originally in Knessl and Peters [15]) and using the same method for the Laplace inversion i.e. the ACM routine 619 with tolerance Tol $2=1 . D-4$ gave maximum absolute error equal to $0.31 D-5$ for $z=2$ and $t=1,2, \ldots, 10$.

The fully numerical treatment of the problem described here and also the fully numerical direct approach of Makroglou [19] which uses global multiquadric approximations, allow the easy extension for other types of distribution function $B(x)$ and premiums function $\beta(Z(t))$ when finding the exact solutions for $R(z, t)$ or the corresponding Laplace transforms of $R(z, t)$ is not easy or possible, and thus they qualify for further investigation and extensions.

\section{REFERENCES}

[1] H. Albrecher, J.L. Teugels and R.F. Tichy. On a gamma series expansion for the timedependent probability of collective ruin. Insurance: Mathematics and Economics, 29, 345-355, 2001.

[2] G. Arfwedson. Some problems in the collective theory of risk. Skand. Aktuar. Tidskr, 33, 1-38, 1950.

[3] I. Bonzani. Solution of nonlinear evolution problems by parallelized collocationinterpolation methods. Computers Math. Applic., 34, 71-79, 1997.

[4] R. Brekelmans and A.De Waegenaere. Approximating the finite-time ruin probability under interest force. Insurance: Mathematics and Economics, 29, 217-229, 2001.

[5] F.E. De Vylder and M.J. Goovaerts. Explicit finite-time and infinite-time ruin probabilities in the continuous case. Insurance: Mathematics and Economics, 24, 155-172, 1999.

[6] D.C.M. Dickson and H.R. Waters. Ruin probabilities with compounding assets. Insurance: Mathematics and Economics, 25, 49-62, 1999.

[7] G. Fairweather. Spline collocation methods for a class of hyperbolic partial integrodifferential equations. SIAM J. Numer. Anal., 31, 444-460, 1994.

[8] J. Grandell. Aspects of risk theory. Springer-Verlag, 1991.

[9] W. Harper. Numerical treatment of the problem of collective ruin. In: Final year project, Dept. of Mathematics, University of Portsmouth, UK, 1995.

[10] W. Harper. On the estimation of the survival probability for the problem of finite time collective non-ruin. In: M.Sc. thesis, Dept. of Mathematics, University of Portsmouth, UK, 1996.

[11] Z.G. Ignatov, V.K. Kaishev and R.S. Krachunov. An improved finite-time ruin probability formula and its Mathematica implementation. Insurance: Mathematics and Economics, 29, 375-386, 2001.

[12] H. Jasiulewicz. Probability of ruin with variable premium rate in a Markovian environment. Mathematics and Economics, 29, 291-296, 2001.

[13] E.J. Kansa. Multiquadrics - A scattered data approximation scheme with applications to computational fluid dynamics-I, Surface approximations and partial derivatives estimates. Computers Math. Applic., 19, 127-145, 1990.

[14] E.J. Kansa. Multiquadrics - A scattered data approximation scheme with applications to computational fluid dynamics-II, Solutions to parabolic, hyperbolic and elliptic partial differential equations. Computers Math. Applic., 19, 147-161, 1990. 
[15] C. Knessl and C.S. Peters. Exact and asymptotic solutions for the time dependent problem of collective ruin. I. SIAM J. Appl. Math., 54, 1745-1767, 1994.

[16] C. Knessl and C.S. Peters. Exact and asymptotic solutions for the time-dependent problem of collective ruin. II. SIAM. J. Appl. Math., 56, 1471-1521, 1996.

[17] X.S. Lin and G.E. Willmot. The moments of the time of ruin, the surplus before ruin, and the deficit at ruin. Insurance: Mathematics and Economics, 27, 19-44, 2000.

[18] A. Makroglou. Computer treatment of the problem of collective non-ruin; the finite time case. In: Contributed talk: 18th International Congress on Computational and Applied Mathematics (ICCAM '98), July 27-August 1, 1998, Katholieke Universiteit Leuven, Belgium, and Conference HERCMA '98, 24-26 September. AUEB, Athens, Greece, 1998.

[19] A. Makroglou. Multiquadric methods in the numerical treatment of the integrodifferential equations of collective non-ruin; the finite time case. In: Boundary Element Technology XIII incorporating Computational Methods and Testing for Engineering Integrity, Proceedings of the 13th International Conference on Boundary Element Technology (BETECH '99), June 8-10, Las Vegas, Nevada, USA. WIT Press, 495-504, 1999.

[20] A. Makroglou. Computer treatment of the integro-differential equations of collective non-ruin; the finite time case. Mathematics and Computers in Simulation, 54, 99-112, 2000.

[21] A.K. Pani, Thomée, V. and L.B. Wahlbin. Numerical methods for hyperbolic and parabolic integro-differential equations. J. Integral Equations and Applic., 4, 533-584, 1992.

[22] J. Paulsen. Ruin theory with compounding assets-a survey. Insurance: Mathematics and Economics, 22, 3-16, 1998.

[23] Jr. Pervozvansky, A.A. Equation for survival probability in a finite time interval in case of non-zero real interest force. Insurance: Mathematics and Economics, 23, 287-295, 1998.

[24] C.S. Peters and M. Mangel. New methods for the problem of collective ruin. SIAM J. Appl. Math., 50, 1442-1456, 1990.

[25] R. Piessens and R. Huysmans. Alg. 619, Automatic numerical inversion of the Laplace transform. ACM Trans. Math. Soft., 10, 348-353, 1984.

[26] H.L. Seal. The numerical calculation of $u(w, t)$, the probability of non-ruin in an interval $(0, t)$. Scand. Actuarial J., 121-139, 1974.

[27] H.L. Seal. Survival probabilities. The goal of risk theory. John Wiley and Sons, 1978.

[28] M. Usabel. Calculating multivariate ruin probabilities via Gaver-Stehfest inversion technique. Insurance: Mathematics and Economics, 25, 133-142, 1999.

[29] H. Yang and L. Zhang. On the distribution of surplus immediately after ruin under interest force. Insurance: Mathematics and Economics, 29, 247-255, 2001.

[30] E.G. Yanik and G. Fairweather. Finite element methods for parabolic and hyperbolic partial integro-differential equations. Nonlinear Analysis, Theory, Methods and Applications, 12, 785-809, 1988.

Integralinès lygtys ir aktuarijų rizikos vadyba: kai kurie modeliai ir skaičiavimai

\section{A. Makroglou}

Sprendžiamas tikimybès $R(z, t)$ ( $t$ - laikas, $z$ - pradiniai rezervai) įvertinimo uždavinys, kur $R(z, t)$ - tikimybè, kad verslo (tarkime, draudimo) bendrovè nesubankrutuos per baigtini laiką $t$. Tuo tikslu naudojami literatūroje aprašyti integraliniai ir integrodiferencialiniai modeliai, apžvelgiami kai kurie analiziniai ir skaitiniai lygčių sprendimo metodai, pateikiami skaičiavimų, gautų vieno metodo globalinès Lagranžo tipo aproksimacijos $z$-erdvėje pagalba, rezultatai. 